\title{
5) Handelsberichte.
}

Stuttgart, den 25. August 1852.

0 st - und Westindische Droguen.

Aloe plötzlich gestiegen auf die Nachricht von der Fortdauer des Kriegs auf dem Cap der guten Hoffnung. Aloe Barbados sehr billig; diese Sorte, bei uns noch wenig im Gange, wird in England zu PferdeArzneien in grosser Quantilät verwendet.

Ambra grisea untersuchte ich den ganzen Vorralh in London und fand zwar echte Qualität, aber von etwas duffem Gerach, der sich verlieren wird.

Arac besitze ich in echter Qualität aus Batavia in ganzen Leggers und im Anbruch in Consumo und auf Transito-Lager.

Balsam. copaivae fehlt auf den meisten Plätzen, weil die Zufuhren bisher so unbedeutend waren, dass sie gleich nach ibrer Ankunft verschwanden. Ich habe meinen Vorrath zusammengehalten, um meinen Abnehmern fortwährend damit dienen zu können. Bals. Peruv. nigr. ver. Die Zufuhren waren in diesem Jahre grösser als sonst. Der Preis ist geblieben.

Banilles feinst krystallisirte, lange von $7 \frac{1}{2}-8 \mathrm{Zoll}$, besitze ich zwar noch, aber in beschränktem Vorrath, und wüsste ich mir keinen neuen anzuschaffen, denn die diesjährige frisch angebrachte, die Manches zu wünschen übrig lässt, braucht bekanntlich längere Zeit zu ihrer Entwickelung.

Cacao besitze ich alle Sorten, den Guayaquill in bester Qualität in grossen Bohnen, die auf den ersten Plätzen fehlt, da wegen der Unruhen im Lande die Zufuhren ausbleiben. Cacao aus Para an gesunder Waare ist billig. Nebst dem echten Carraceas von Porto Cabello habe ich auch den ebemals so beliebten fetten Surinam, der aber gar nicht mehr regelmässig in Handel kommi, weil dessen Anbau durch Caffeeplantagen verdrängt wurde. Auf Java hat man auch angefangen Cacao zu pflanzen, von welchem mir ein kleines Nuster zugesandt wurde, worüber sich kein Urtheil fällen lässt.

Cardamomen Ceylon'sche und Malabar sind auf ihrem niedrigsten Standpuncte angekoinmen.

Castoreum von Canada und Hudsonsbay lsann bei allmälig abnehmenden Zufuhren nicht billiger werden. Es ist ein wahrer Hohn, dass noch immer unter der Benennung Bayrisches, Polnisches oder Moscowitisches Castoreum, welche beide allerdings kaum noch zu belommen sind, eine Sorte in Handel herumschleicht und a 20 . fl. circa verkauft wird, die nur aus dem Amerikanischen besteht, von welchem die hiezu nach Form und Grösse geeigneten Beutel mamputirt werden, die aber weder den intensiven Geruch, noch das Zellengewebe besitzen wie das echte.

Canehl Ceylon ist auf seinen niedrigsten Preis gekommen, der aber bereits wieder anzuziehen begonnen hat. Die Aulhebung des früheren sehr hohen Ausgangszolles und die Rivalität des Javazimmts haben die erwähnte Preiserniedrigung hervorgebracht. Von

Cassia lignea Zimmt in kleinen Bündeln sind die Vorrathe in Europa sehr zusammengegangen. Das letzte aus Canton in England angekommene Schiff brachte nur 100 Kisten. Im Jahr 1846 betrugen die Vorräthe in England 30,000 Kisten. In Canton waren die Schiffe nach Europa rar und die Frachten hoch, weil die Chinesen in Massen 
nach den Goldländern auswandern. Die eigentliche Consomtionszeit in Europa und die Verschiffungen zur Versorgung des Nordens finden in den nächsten Monaten statt. Nachrichten aus Canton vom 21. Juni bringen den Preis von 18 Dollars, wozu für England Einiges gemacht wurde, indem die Chinesen sich nur langsam bequemen, ihre Forderungen zu ermässigen. Von meinen vor drei Monaten in Hamburg eingekauften 500 Kisten bleiben dort noch 120 Kisten übrig, - Flores Cassiae sind sehr rar und hochgehalten.

Chinarinden. Es feblt nicht an der Monopolwaare, die aber in den Händen der reichsten Häuser ist, welche mit grösster Zähigkeit auf hohe Preise halten. Wenn Sie von dieser Sorte bedürfen, so kann ich Ihnen die echte platte ohne Rinde, und die in Röhren init der $R$ inde, welch letztere selten ungemischt im Handel vorkonmt, in ausgewählten Stücken ohne Bruch und Gras zutheilen. Hein Vorrath in allen den verschiedenen Sorten von gelber und grauer China besteht selten unter 300 Surons, so dass mir eine Auswahl zu Gebote steht, wie sie nicht überall angetroffen wird. Die Verschiedenheit der Preise und Qualitáten macht es mir wünschenswerth, auf Muster zu verkaufen, mit denen ich gerne zu Diensten stehe.

Cubeben scheinen auf den niedrigsten Stand gekommen zu sein.

Fabae tonco finden sich nur in beschränkter Quantität.

Gummata a s 0 stindien und Afrika.

Gummi Ammoniae wird nicht mehr sn stark begehrt wie fruher; an Asafoetida kein Mangel.

Gummi Benzoe ist in den mittleren Sorten sehr billig geworden, die fein mandolirten erhalten sich im Werthe.

Gummi Copal. Man darf sich nicht wundern, dass derselbe in allen Sorten so theuer geworden ist, denn es sammeln sich keine Vorräthe an den Bezugsquellen, und was dort ankommt, wird begierig mit höheren Preisen aufgekauft. So lange diese Verhältnisse sich nicht ändern, wird derselbe rar und theuer bleiben. Es pressirt mir daher nicht mit dem Absatz meines Afrikanischen harten schmelz- und schleifbaren blank gewaschenen Copals. Was den Brasilianischen und Westindischen gelben harten mit dunkeln Stücken und 10 Proc. Grus betrifft, so erhalte ich heule die Anzeige von dem Verkaufe eines Postens in Hamburg à 8 f Schilling Banco, während ich denselben hier wohlfeiler abgebe.

Gummi Dammar begehrt und gestiegen; Elemi sehr billig; Elasticum fehlt in kleinen Beuteln, bei den grossen Beuteln richtel sich der Preis je nach der Feinheit 'der Pasta und ob sie hohl oder voll sind. Von dem in gewalzten Blättern habe ich immer Vorrath in verschiedenen Dimensionen. Von dem bjlligen Batavia Kautschuk, der auch stark gebraucht wird, habe ich am Wege. Gummi Guttae war nie so billig als jetzt; Kino schönste Waare zu einem Spottpreise; Olibanum wird fortwährend für Russland aufgekauft. Schellack habe ich in feinst gelber Qualität, die aber früher öfters decidirter vorkam als jetzt, wo dieselbe so selten ist, als die mittleren Sorten genug vorhanden sind.

Lign. Quassiae Surin. dünnes ganz und geraspelt sehr gewichen; Sassafras fehlt, ich habe davon mehr geschnitlenes als ganzes; echten Fernambuk am Wege.

Macis und Macisblüthe in Holland gestiegen; die Anfangs nächsten Monats stattfindende Auction wird die künftige Preisbestimmung bringen. 
Moschus ist genug vorhanden, allein die feinste Qualität in vollen ungenähten Beuteln ist nicht so leicht zu finden. Es kam kürzlich eine Qualităt ex vesic. vor, die à 20 f. per Unze verhauft wurde.

Nelken Amboina sind gesuchter und etwas höher.

Diverse Artikel.

Cajeput-Oel hat sich seltener und theurer gemacht; Croton-Oel echt und billig; Ricinus-Oel kommt in ungeheurer Henge und in eben so steigendem Verbrauch, hat aber seinen niedrigsten Stand erreicht. Sternanis-Oel in lelzter Zeit viel zugeführt; Zimmtblülhen-Oel ist im Anziehen; Zimmt-Oel aus Java ist billig, auch das von Ceylon ist wohlfeiler geworden. Dieses letztere, dünkt mich, sei frúher feiner vorgekommen als jetzt.

Orlean Cayenne selten und gestiegen; ich habe davon nur noch weniges ausser Bast in schöner Qualitât.

Pfeffer weisser und langer, beide gestiegen.

Radix Jalappae. Es bestätigt sich, dass vor drei Jahren zu viel ausgegraben wurde, weil alle neueren Zufuhren in sehr kleinen länglichen Wurzeln bestehen, bei welchen die grösseren runden schweren fehlen. Nein Vorrath besteht noch in einer âlteren Waare, die zwar nicht frei von Tadel, aber doch besser ist, als sie gegenwärtig vorkommt.

Radix Ipecacuanhae. Die früheren sehr niedrigen Preise haben in Brasilien das Einsaumeln eingeschüchtert, es kommen nur kleine Parthien und der Preis soll in Rio 2 Dollars per Pfund sein.

Radix Ratanhiae. Die lange erwartele Zufuhr besteht in einer apocryphen Waare, was sehr zu bedauern ist, weil keine gute mehr vorhanden ist. Dagegen besitze ich aber einen Extract, der nichts zu wünschen lãsst, nur etwas höher einsteht als der gewöhnliche im Handel vorkommende.

Radix Rhei Moscowit. Nach Berichten aus St. Petersburg ist die Waare von den besseren Jahrgängen geräumt und man wird erst später von neueren wieder schöne Waare erhalten. Ich habe mir von jener noch einen Vorralh gesichert, womit ich entsprechend dienen kann.

Radix Chinesische. Die grossen Verluste auf alle seitherigen Einsendungen und die Gewissheil, dass in Canton sich keine gut getrocknete tadellose Ware befindel, haben die Zufuhren bedeulend reducirt und die Vorräthe in Europa, die fast nur aus Mitteigattungen bestehen, sehr gelichtet. Es ist anzunehmen, dass die Saison zur Verschiffung dieses Artikels in China vorüber und mithin keine Aussicht vorhanden ist, mit besserer Waare sobald versorgt zu werden. Ich besitze hier und auf auswärtigen Lagern noch Vorrăthe, dic mit jedem andern Markt in Preisen und Qualitäten concurriren, sowohl an runder als platter, welch letztere fast überall verschwunden ist, von der ich noch Einiges an schöner $\frac{3}{4}$ mundirter Qualitât besitze, wie solche nicht leicht anderwärts mehr angetroffen wird. haben.

Radix Senega rüumt sich; Salep ist deutsche und levantische zu

Rhum Jamaica vorräthig. Feinsten ganz starken, den ich seit 12 Jahren am Lager habe, ist noch in Flaschen vorrälhig.

Safran spanischen in breiten Blättern mit wenig gelben habe ich in schönster Qualităt direct aus dem Lande bezogen und glaube aus den seit längerer Zeit entbehrten und neuerdings nach einander gefolg- 
ten Aufträgen schliessen zu dürfen, dass der Artikel in besseren Werth kommt als bisher, weil die Zufuhren stocken. stand.

Salpeter ostindischer und raffinirter treten in einen stabilen Preis-

Sanguis draconis feinsten in Bast habe ich nicht mehr als eine Kiste erhalten kơnnen.

Semen Anisi stellati. Die nicht bedeutenden Zufuhren fanden überall erschöpfte Lager, daher der Preis hoch gehalten wird.

Semen Cynae levant. Trotz dem grossen Verbrauch für Santonin ist der Artikel so schōn, frisch und so billig zu haben, als selten zuvor. rath.

Semen Staphid. agr. sehr rar. Von Stinci marin. habe ich Vor-

Tamarinden sind auf einen Stand des Unwerlhs gekornmen.

\section{Von The e's}

besitze ich alle dic gangbarsten Sorten, von denen ich auch bedeutende Lager im Auslande unterhalte und durch Einkäufe in Quantitaten die Preise auf das Niveau mit grossen Plätzen stellen kann. Wie alle chinesischen Artikel verdient Thee besondere Aufmerksamkeit in Rücksicht des etwa prekären Zustandes dieses Landes.

\section{Levantische Artikel.}

Folia sennae alex. naturell. Vor drei Monaten knufte ich in Triest zwei Ladungen, die mit zwei Schiffen aus Alexandrien dahin kamen, welche zusammen nach dem Ausschiessen von geringen Ballen 2110 riginalballen ausmachten. Es ist ganz frische grüne nicht manipulirte Waare, die weder viele Stiele noch Bruch enthält, wie sie selten angebracht wird. Es bleiben mir noch circa 70 Ballen à 3 Centner, wovon ich, so lange Vorrath in Triest, à $20 \mathrm{fl}$. bei einzelnen, a $18 \mathrm{fl}$. Bank-Valuta bei 10 Ballen abgebe. - Nit ostindischen, Necca bin ich ebenfalls versehen. - Fol. Senna Tinavelli sind in letzter Zeit etwas billiger geworden.

Gallus, schoorzer. Mit meinem Vorrath werde ich knapp ausreichen, bis von der neuen Ernte Zufuhren ankonmen, die überall erschöpfte Láger finden, daher die Preise nicht so schnell zurückgehen werden.

Gummi Arabic. tart. Neuerdings in Alexandrien gestiegen, in Folge dessen die Preise auf Seeplätzen im Anziehen sind. Ich erhalte so eben Nachricht von der Ankunft einer directen Parthie in Antwerden, die ich nach dem Norden gehen lasse.

Gummi Mastix levantiner elect. Ich bin an dem letzten Originalfasse. Von Smyrna verlautet Nichts, dass von dorther Etwas zu erwarten wăre, weil nach früheren Berichten die Sträuche noch Jahre gebrauchen, bis sie wieder eine Ernte liefern.

Gummi Myrrhae habe ich in Qualitaten und in Preisen, die jede Auswahl und Billigkeit gestatten.

Gummi tragacant. in Blättern levantiner elect. räumt sich an der Quelle und wird nicht wohlfeiler.

Opium von Smyrna. Die Ernte von vorigem Jahre ist vergriffen die heurige war sehr reichlich. Demungeachtet ist der Preis kaum nennenswerth gewichen, denn der Verbrauch nach China nimmt oft $400 \mathrm{Kisten}$ auf Einmal weg. Das neue 0pium ist aber immer so frisch und weich, dass überall wegen Eintrocknens Anstände zu befürchten sind. Das sogenannte Thebai'sche ist trocken, aber nicht so reich an 
Morphium wie jenes, dagegen auch billiger, wovon ich häbschen Vorrath besitze, so wie auch von dem Aegyptischen.

Italienische, Sicilianische und andereaus dem südlichen Europa kommende Artikel.

Borax, Toskanischer in England raffinirt und monopolisirt gestiegen.

Cantharides. Ans Russland und anderen Gegenden haben wir bisher nur $z$ weijährige Fliegen erhalten. Wenn nun die heurige Ernte gering ausfallt, in welcher Richtung sich alle Berichte aussprechen, worüber wir innerhalb vier Wochen klar sehen werden, dann steht eine bedeutende Erhōhung bevor. Alle meine Anfträge auf neue Fliegen wurden nur theilweise ausgeführt.

Essenzen Ol. Aurantior. amar. of dulc. Bergamolt, de Cedro habe ich in ersten besten Qualitäten und auch Vorrath von Cedro im Norden lagernd. Letzteres ist gestiegen und wird noch theurer werden, wenn - wie es heisst - die Zitronen nicht gerathen. Oleum Neroli, die Blũthe hat dieses Frühjahr durch Frost noch weit mehr gelitlen als im vorigen Jahre, so dass ich nur mit Yühe einen Vorrath von ellichen und 20 Pfund zusammenbrachte, anch nur ein kleines Quantum von heuriger Pomeranzenblüthe. Oleum petils grains besitze ich in vortreffticher Qualität; Oleum lauro cerasi durch Uebernahme einer Parthie billig.

Rosenäl, türkisches. Für meine Qualität kann ich bürgen, dass sie ganz echt ist, die ich aus erster Quelle in Cannister von Zinn erhalte. Oleum Palma rosa, Ol. Geranii besitze ich ebenfalls.

Mandeln. Puglien hat in diesem Jahre einen Ausfall von 40,000 Canturi. Nach den Börsen in Bari und Neapel richten sich die Preise in Sicilien. In Frankreich sind die Aussichten gut, doch bereits viele Lieferungsverträge abgeschlossen. In Triest ist der Vorrath gegenwärtig gleichsam null. Eine Partie süsser Puglieser Mandein von 1851, die für mich in Rotterdam angekommell, disponire ich theils nach Mannhein theils nach Altona.

Manna. Die anticipirten Berichte aus Sicilien, auf welche sonst nicht viel zu halten ist, stimmen aber alle dahin überein, dass es des günstigsten Wetters im ganzen August bedarf, um eine Raccolta weit unter einer mittelmässigen erwarten zu dürfen.

Oleum Jecoris nselli rothblank und gelbblank von heuriger Sammlung habe ich erhalten und davon in Cöln und Mannheim und hier am Lager. Es sind in diesem Jahre grosse Aufträge nach Bergen aus Ländern eingegangen, welche diese Medicinalthransorten früher nie bezogen haben Der gelbblanke ist bereits an der Quelle vergriffen. Für etwaigen Gewichtsabgang unterwegs durch Ausschweissen der Originaltonnen kann ich nicht einstehen.

Oleum, Neufundländerthran habe ich ebenfalls in frischer, heller Waare, wovon der Preis bei Fass von 400 Pfund circa noch ermässigt werden kann.

Oliven-Dele sammtlich im Steigen.

Succus liquiritiae Calabreser $P S$, welche Marke seit mehreren Jahren mit Beifall aufgenommen wurde, habe ich 40 Kisten nach Antwerpen gehen lassen, von denen ein Theil in Cöln bleibt.

Deutsche Vegetabilien.

Es lässt sich zwar noch keine vollständige Uebersicht über*die Ergebnisse der heurigen Sammlungen geben, weil noch Lieferungen 
ausstehen; allein über Chamillen wiess man, dass sie aller Orten gefehlt haben, so dass ich mil aller Mühe nur ein beschranktes Quantum erbalten konnte, wobei ich es im Preis und Qualitãt nicht genau nehmen durfte. Von Römischen Chamillen habe ich von erster Pflācke erhalten; sie sind durch die grosse Hitze schnell zur Reife gelangt, dadurch aber nicht so gross geworden und enthalten auch etwas braune. Herba Absynthii ist viel bei uns gesammelt worden; das daraus destillirte Oel etwas billiger. Von Herba Belladonnae, Digitalis, Galleopsid. wird das Erstere viel mehr verlangt, als aufgebracht werden kann; Herba Menthae, Melissae wird viel angeboten; beide Kräuter werden hier zu Land ganz in Blätter ohne Stiel gesammelt. (In Amerika ist die Pfeffermünze missrathen und das Oel dort theuer geworden.) Wollblumen haben durch die Hitze und nachher durch Regen gelitten. Radix Altheae wird bis zur nächsten Ausgrabung sich auf böherem Preise halten. Secale cornut. unwerth, fehlt an Absalz. Anis, sächsischer soll ganz missrathen sein (es dürfte daher das russische Anisöl sehr Beachtung verdienen). Semen lycopodii wird erst gesammelt; älterer Vorrath nicht gross.

\section{Chemikalien.}

Acid. ritric. Aller Citronensaft wurde aufgekauft und in eine Hand gebracht, das Quantum war aber nicht gross, daher haben die Fabrikanten des Salzes Schwierigkeiten, sich dasselbe zu verschaffen, und weil der Begehr nach letzterem so stark ist, müssen die Lieferungen voraus bestellt und die geschraubten Preise bewilligt werden.

Acid. lartar. ist schön und sehr billig.

Chinin. Der Begehr komrat von allen Seiten; alle Fabriken können kaum die Bestellungen befriedigen. Rücksichtlich des Preises folge ich stets den Verbältnissen, ebenso mit den andern Chinin-Präparalen, so wie mit Chinoidin in Tafeln. Cinchonin blendend weisses sehr billig.

Jodkalium feinst weiss krystallisirt und trocken richtet sich nach dem Preis des Jods, das in England vorerst nicht gestiegen ist, weil der Hauptbedarf ersi später eintritt. Doppelt sublimirtes Jod stets vorräthig.

Morphium hält sich, so lange der Preis des Opiums nicht billiger wird.

Phosphor, amorpher. Die vollständige Befreiung von nicht amorphen scheint noch nicht gelungen zu sein.

Santonin. Es ist zweifelhaft, ob ferner zu dem so sehr herabgedrückten Preise verfertigt werden lsann.

In meinen Preisen der äbrigen Präparate sind keine wesentlichen Veränderungen vorgefallen.

Folgende Artikel verdienen schliesslich noch einer besonderen Erwàhnung :

Mercurius vivus. Die ersten 1000 Flaschen aus Californien sind in England eingetroffen und treten in Concurrenz mit dem Spanischen und Oesterreichischen bei Annahme grosser Parthien.

Terpentin venez Wird sich in Kurzetn zeigen, ob die heurige Fechsung besser gerăth als die vorjährige, die keine klare Waare geliefert hat.

Terpentin-Oel. Ich erwarte jeden Tag die Nachricht von der Ankunft in Hotterdam von 50 Fässern à circa 400 Pfund, die theilweise nach dem Norden und nach Mannheim bestimmt sind. 
Grünspan. In Folge des bedeutenden Steigens des Sprits in Frankreich gestiegen.

Kusso kommt hinfüro nur in Bündeln mit den Stielen vor, der aber ungleich billiger ist als der in Blättern. Nach einem Bericht rom 20sten dies, aus Hamburg wurde $\frac{1}{2}$ geschalle $R$ habarber, was von guter Waare zu finden war, à 30 Schilling Banco aufgekauft.

Ich wünsche, dass dieser Bericht freundlich aufgenommen werde, der von dem heutigen Stande der Handelsverhältnisse in möglichst genauem Zusammenhange mit grossen Plätzen verfasst ist, in dieser Beziehung eine kurze Uebersicht darbietet von Dem, was auch auf einem kleinen Platze geleistet werden kann, um nicht von den grossen verdrängt zu werden, womit ich meine Dienste empfehle und mit aller Achtung beharre

$$
\text { Friedr. Jobst. }
$$

\section{Herbst - Bericht.}

Hamburg, den 1. September 1852.

Der IIandel war diesen Sommer im Allgemeinen ziemlich befriedigend, und zweifeln wir auch nicht, dass das Herbstgeschäft recht lebhaft und umfangreich wird; aussergewöhnliche Conjuncturen baben bis jetzt nur einige Artikel, die dem Colonialfache ungehören, erlitten, und zweifeln wir, dass irgend eine Drogue sich solcher Conjunctur unterwirft, da unser Platz bedeutende Vorrăthe erweist und auch unser Lager als wohlassorlirt zu betrachten ist, was uns in die Lage setzt, jede Entbietung in der lsürzesten Zeit aufs beste zu effectuiren.

Nun einige Bemerkungen über die hauptsächlichsten Droguen in mõglichster Kürze zusammengefasst.

Acid. citric. cryst. wird das Engl, in schönen weissen runden Krystallen dem Franz. Prima vorgezogen, da ersteres trockner, weisser und hübscher krystallisirt.

Agaricus alb. mund. erwarten wir pr. "Ernst August ", Capt. G H. Kramer, von Archangel, 12 Fässer, ca. 4000 Pfd., die billig einstehen.

Aloe succotrina wieder in schöner Qualităt erhältlich, was wohlthuend auf den Markt einwirkt.

Ambra grisen, beste schiefrig brechende Waare und fein von Geruch etwas billiger.

Anacardia occidenlalis tindel in neuer Zeit wieder mehr Anwen. dung und dienen wir, so lange Vorrath, mil frischester Waare.

Aqua lauro cercisi vera, von frisch gepflückten Blättern bereitet, können zu der niedrigen Notirung empfehlen.

Arrow-rool Bermudas, in echter Waare, in den beliebten Original-Blechdosen von ca. 30 Pfd, erfreut sich lebhaften Absatzes, und das nicht mit Unrecht, denn obgleich der Preis gegen die andern geringeren Sorten hoch zu nennen ist, so giebt doch die gute anerkannte Qualitāt reichlichen Ersatz dafür. - Von

Baccae lauri, gesiebt, war unser Markt einige Zeit ziemlich entblössı, doch haben wir kürzlich pr. "Adagio “, Capt. H. Eggers, Einiges von Triest erhalten und können mit schöner Waare dienen.

Balsam. copairae ist beinahe der einzige Artikel, der Schwierigkeiten macht, ihn in genuigender und echter probehaltiger Waare zu 
erhalten und muss man dafür höhere Preise bewilligen; - de peruv. ver, nigr. ist wieder hinlänglich in besler Waare zu baben und die Preise dafür nicht überspannt, es sind ca. 2000 Pfd. jüngst pr. vMargaretha « von Amapala angebracht, die in Auction kommen dürten.

Camphor raffin., dieser früher so beliebte Speculationsarlikel liegt seit laingerer Zeit beinahe ganz danieder und wird zu Unternehmungen von einiger Bedeutung nicht passend gehallen; die Zufuhren von rohem Camphor treffen von Canton zu regelmässig und in ziemlich bedeutendem Maasse in allen Seeplätzen ein, so dass der Preis, ausgenommen einige unbedeutende Erhöhungen, die durch augenhlickliche vermehrte Nachfrage veranlasst, sich immer gleich bleibt. - Von

Cantharides räumten sich die alten Vorräthe zu steigenden Preisen in letzter Zeit rasch auf, da Berichte von sehr geringen Einsammlungen in Ungarn und der Wallachei bekannt wurden, und sind in Folge dessen, wie man uns aus Russland schreibt, Ordres aus beiden erstgenannten Productionsländern in Russland eingetroffen. Eine seltene Erscheinung, die jedoch nicht dazu beiträgt, uns billige Preise erwarten zu lassen, und dürfte unter solchen Umstãnden eine fernere Steigerung dieser Fliege unausbleiblich sein. - In

Cassia lignea haben unsere Zufuhren an Bedeutung und Umfang zugenonımen und waren unsere Preise unter Englischen und Holländischen Mărkten, so dass von daher Ordres einliefen; es blieben die Preise dem ungeachtet ziemlich stabil bis vor ca. 3-4 Wochen, wo ein biesiges Haus sich auf diesen Artikel warf und auf allen Plätzen von einiger Bedeutung beträchlliche Aufkäufe machle, und so eine Steigerung von ca, $10-15$ Proc. hervorrief, weshalb vorerst keine Aussicht auf einen Rückgang; - vera ist in Folge dieser Steigerung auch etwas besser bezahit.

Casioreum canadens. besilzen in schönen vollen Beuteln, das seines ausgezeichneten Geruches wegen beslens empfehlen können. Sehr wenig Bedarf zeigte sich von

Chinin sulphuric. gallic. prima albiss. Da sich die sonst im Herbst gewöhnlich eintretenden Fieber-Epidemien glücklicher Weise nicht einstellten, so konnten wir den Preis dafür etwas ermässigen, was jedoch von keiner langen Dauer sein dürftc, da die Vorräthe von gehaltreicher Chinarinde unbedeutend zu nennen sind und die neuen Zufuhren immer mehr und mehr mit weniger gehaltreicher und gar unächter Rinde vermischt hier eintreffen, was, wenn auch momentan, doch nach und nach einen Einfluss auf die daraus zu bereitenden Präparate ausüben dürfe, - Gut assortirl ist unser Markt von

Cortex chinae, da wir im Mlonat Juli pr. "Victoria" von Payta bedeutende Zufuhren, ca. 200 Kisten, hatten, die in Auction gebracht wurden, wobei wir uns sehr stark betheiligten und mit bester huanoco opt., gut grau bis silbergrau, theilweise tief chagrinirte Röhren von $\frac{1}{1}-\frac{3}{4}$ Zoll dick, loxae, theilweise mit Noos besetzt, hübsch im Bruch und frisch von Geruch, und miltelstarke Röhren, so wie regia cum el sine opid. in schönster Qualität, dienen können. - Die günslige Witterung dieses Sommers ist dem

Crocus gastinois sehr zuträglich gewesen und lauten die Berichte aus ganz Frankreich, dass eine günstige Ernte zu erwarten ist. Wir haben in Folge dessen unsere Notirung bereits ermässigt und werden nach Eintreffen neuer Waare, ungefähr im Monat October, wenn möglich eine weitere Preisermässigung eintrelen lassen. 
Cubebae sine slipid, die wir selbst elegiren lassen, verkaufen sich flotl zu billigen Preisen.

Flores rosar. rubr. nov. opt. lieferte die jüngst beendigte Einsammlung eine gegen frühere Jahre geringe Ausbeute, die Qualită ist jedoch hübsch und halten die Landleute noch hoch danit; es wird hauptsächlich von den einlaufenden Ordres abhängen, wie sich die Preise für die Folge gestalten; - chamomillae vulg. wurden wenig gesammelt und die Frage danach war ziemlich stark, so dass der Preis etwas Faveur nahin; - roman. werden uns leider auch in diesem Jahre in nicht ganz untadelhafter Qualität geliefert, da die grosse Hitze während der Blüthezeit die Blumen theilweise auf dem Stocke bräunlich brannte und der Mangel an Regen sie nicht zur vollkommenen Grösse auswachsen liess; wir waren jedoch bemüht, uns von den in diesem Jahre am schönsten fallenden Blumen erster Blüthe zu verschaffen, und dienen damit, so lange Vorrath, zu civilen Preisen.

Fol. sennae alexandr. erhielten neue Zufuhren aus Triest in schöner grüner frischer Waare, gut von Blatt, die billig einstehen.

Gallus Aleppo mussten wir alle Sorten erhöhen, der fortwährende Verbrauch lässt keine Lager entstehen, und dürfte dieser Steigerung vor Eintreffen neuer Waare kein Einhalt zu thun sein.

Gelatinae wird nun auch in schōner carninrother Waare erzeug1, die jedoch etwas höher im Preise als die dünne weisse und durchsichtige Waare. - Von

Gummi benzö̈ bietet unser Markt in schöner amandolirter, so wic Secunda- und Tertia-Waare schỡne Auswahl; - copal Ostind. fehlte lange Zeit beinahe gänzlich und wurden die jüngst versteigerien 53 Kisten diverser Qualităt hoch bezahlt; - Westind. nat ebenfalls sehr rar und bessere Preise bewilligt, doch setzt uns hinlänglicher Vorrath in den Stand, Sie die billigeren Preise geniessen zu lassen; - damar. elect. albiss. ebenfalls höher, und scheint es, als ob die Gummata, die irgendwie grössere Verwendung zu technischen Zwecken haben, an Verbrauch zunehmen; - guttae in Röhren ist billig zu nennen für die hübsche Qualität; - lacca in tabulis bietet unser Markt fortwährend gute Auswahl in ff. orange, f. med, leberfarben und braun; in dicken Blältern, hier unter dem Namen "Blutscheilack " gangbar, erwarten wir pr. Schiff $*$ Alma \& Olga , Capt. Meyer, direct von Calcutta 50 Kisten in drei Nüancen, $d$. $i$. f. orange in noch nie dagewesener schōner Qualităt, dann f. orange und braun; - sanguis draconis $\mathrm{ff}$. Waare in Schilf, so wie in Massa erhielten neue Zufuhren von ausgezeichneter Schönheit, ebenso f. med. in Massa in $\square$ Tafeln sehr empfehlenswerth.

Gutta percha in Prima-Waare bei grosser Auswahl billig zu kaufen. - Von

Herbas sind beinahe schon alle in neuer Waare eingetroffen; belladonnae wie gewöbnlich in verschiedener Qualität geliefert, wonach sich der Preis richtet; - cardui benedicti verhältnissmässig wenig und in nicht sehr schöner Qualität geliefert; ${ }^{-}$menthae pip. et crisp., so wie melissae in foliis sind in Folge der wohl etwas übertriebenen Berichte der Cholera etwas fester im Preise; wir hoffen jedoch, dass diese jetzt beinahe jährlich wiederkehrende Seuche nicht so verheerend auftreten und dann wohl keinen grossen Einfluss auf die als wirksame Gegenmittel anerkannten Kräuter und andere Waaren ausüben wird; - palschuli in frischer parfümreicher Waare Kleinigkeiten an den Jarkt gekommen. - Von 
Hydrarg. virum sind zum ersten Male 1000 Flaschen von San Blas, Californischer Mine, angebracht, und dürfte diese ziemlich bedeutend zu nennende Zufuhr etwas rückgängige Preise bringen, denen dann auch bald die daraus erzeugten Präparate folgen werden.

Jodine gallic. in schönster breitbläıriger trockner Waare, so wie angl., klein und unansehnlich gegen gallic., ist billig, bleibt jedoch ohne Beachtung.

Kali chrom. rubr. Nordisches so wie Englisches bleibt angetragen und billig käuflich; - hydrojod. anglic, sicc., so wie gallic. albiss. konnten in Folge der billigen Jodpreise etwas ermässigen und kōnnen zu Beziehungen nur einladen; - oxymuriat. in Schuppen billig zu kaufen.

Kreosot. in wasserheller Waare hönnen in Folge grösserer Entbietungen von den renommirtesten Fabrikanten billiger erlassen.

Lactucarium german. Der getrocknete Milchsaft aus der Pfanze Lactuca virosa findet in der Medicin immer mehr Anwendung, und können wir Ibnen dasselbe, von einem gewissenbaften Apothelier bereitet, in echter reiner und unverfälschter Waare zu billigen Preisen anbieten.

Manna ist in Sicilien, so wie anf dem Hauptmarkte Genua gänzlich geräumt, und reichen unsere Vorrãthe kaum bis zur neuen Sammlung, worüber sich jedoch nichts Bestimmtes sagen lässt, obgleich Sachkundige behaupten wollen, dass dieselbe nicht ergiebig ausfallen wird, da die Manna-Esche zu stark in die Blüthe getrieben haben soll. - Die

Morphium-Präparate müssen wir etwas im Preise erhöhen, da Opium in Constantinopel bedeutend im Preise anzog.

Ol. bergamot. de cedro et Porlug. besitzen in echten feinsten Qualitaten und sind die Aussichten der bevorstehenden und theils begonnenen Ernte gut. Der Einkauf von $O l$. berg. erheischt jedoch die grösste Vorsicht, da die neue Methode eingeführt, mit einer Maschine die Essenz auszupressen, auf welchem Wege man grössere Quantitäten als durch Auspressen mit der Hand erzielt; wir geben jedoch der durch Auspressen mit der Hand gewonnenen Essenz den Vorzug, da sie von feinerem Geruch und von schöner gelblicher Farbe; - anisi stellati erhielten neue Zufuhren und konnten wir die Notirung etwas reduciren; - von jecoris aselli lauteten die Berichte aus Bergen vom Fruhjahre, dass die Fischerei zwar gũnstig, allein was das Quantum Thran, besonders album anbetrifft, wird dasselbe, indem die Leber eben so mager wie voriges Jahr, nicht grösser werden, und darf man bei dem Mangel an Vorräthen so wie dem zunehmenden medicinischen Bedarfe, auf billige Preise keine Rechnung machen; dies traf denn auch ein: da sich die Aufträge für die ersten Zufuhren zusammendrängten, kauft man jetzt, wo wir unseren Bedarf deckten, billiger, und sind in jeder Sorte gut assortirt; - ol. olivar., alle Sorten elwas fester; - ricini erwarten wir pr. -Alma \& Olga a, Capt. Meyer, von Calcutta 100 Kisten in der belieb. ten Packung à 4 Blechdosen von ca. $40 \mathrm{Pfd}$. pr. Dose, in bester frischester weissester Waare, Probe davon erhielten wir bereits pr. Oberland-Mail via Triest, die Nichts als den Wunsch übrig lässt, die Waare möge genau dem Muster entsprechen.

Orlean. brasil., Prima feurig, erwarten pr. "Especulador", Capt. de Rocha, 80 Körbe.

Opium thebaic. opt. ist, wie bereits unter Morphium erwähnt, bedeutend im Preise gestiegen, es sind ca, 300 Kisten zwischen Smyrna 
und Constantinopel an den Markt gekommen, die flott zu steigenden Preisen für England, Amerika und China Nehmer fanden; wir erwarten wieder eine Kiste der beliebten Guévé-Sorte.

Rad. althea verspricht eine ergiebige Ernte, die jedoch erst im Spăt-Herbste begiunt; - alcanna wieder reichlicher aus Ungarn nun in schöner electer blüthenfreier Waare zugeführt; - angelica in $Z$ öpfen gefochten, sehr billig notirt; - calami mund. alb. lebhaft gefragt und höher erwartet; - gentiana rubr. ist niedrig und bezahlt man die Einsammlung nicht mehr so, dass ein Aufschwung der Preise in Aussicht steht; - von jalappae pond. Irafen wieder grössere Zufuhren ein, aber grösstentheils in etwas länglicher Wurzel, die doch resineuse sind, und konnten wir im Preise etwas Soulagement gewähren; ipec. ist hier ausgekauft, und wenn uns nicht von den pr. letzlen Steaner von Rio Janeiro nach England gebrachten 53 Seronen Einige zukommen, werden wir bei Bedarf überspannte Forderungen bewilligen müssen; - liquirit. hispanic. so wie russ. mund. in guter Auswahl; - rhei moscowit. besitzen Waare der letzten Jahrgänge sehr hübsch; ostind. $\frac{1}{2}$ in und. elect. und rund bleibt begehrt; - von ratanhiae sind schöne dichte lange Wurzeln selten und theuer, dagegen dünne mit etwas Knollen versehene Waare billig; - sassapar. Costaric., die wir der Erwâhnung werth halten, da diese Sorte jetzt nach und nach in Aufnahme konmt; sie gleicht der Caraccas-Sorte, ist jedoch elwas dicker und die äussere zarte Oberrinde röthlich; valeriana harz. so wie minor trafen in neuer und befriedigender Ware bereits ein.

Semen anisi vulg. soll die Ernte als eine spärliche anzusehen sein und wurden in Folge dieser Nachrichten bereits einige Speculationskäufe gemacht, die die Preise etwas befestigten; - cydonior. elect. erhielien aus St. Petersburg billige und schöne spatenfreie Ware; lycopodii bleiben geringe Vorräthe, womit wir uns bis Eintreffen des neuen Sorumers behelfen müssen.

Spermaceti albiss. anglic. etwas billiger zu erlassen.

Spongiae marinae levant. elect. bleiben schwer erhältlich, wir notiren, so lange unser Vorralh reicht, die niten Preise.

Succus liquiril., können die Sorte in dicken Stangen von reinem Geschmack, mit dem Fabrikstempel Cassano, empfehlen.

Tamarinden. Ostind schwarz und sauer, mil móglichst wenig Kernen, ermãssigt in Yreise. - Obgleich von anderen Plätzen eine Steigerung von

Vanillac gemeldel warde, so dienen wir doch noch mit fetter schwarzer krystallischer Waare in allen Lãngen zu den billigsten Preisen. - In stabil.

Zincum melallic, wenig Umsalz und bleiben die Preise ziemlich

Wir halten uns Ihren Diensten in jeder Beziehung hereit und wünschen nur, dass Sie dieselben recht hãufig über uns verfügen.

Mlit aller Achtung ergebenst

Schubart of Bade. 